\title{
Reactive Oxygen Species Induce Epigenetic Instability through the Formation of 8-Hydroxydeoxyguanosine in Human Hepatocarcinogenesis
}

\author{
Naoshi Nishida a, b Tadaaki Arizumi ${ }^{a}$ Masahiro Takita ${ }^{a}$ Satoshi Kitai ${ }^{a}$ \\ Norihisa Yada ${ }^{a}$ Satoru Hagiwara ${ }^{a}$ Tatsuo Inoue ${ }^{a}$ Yasunori Minami ${ }^{a}$ \\ Kazuomi Ueshima ${ }^{a}$ Toshiharu Sakurai $^{a}$ Masatoshi Kudo ${ }^{a}$ \\ a Department of Gastroenterology and Hepatology, Kinki University School of Medicine, Osakasayama, and \\ ${ }^{b}$ Department of Gastroenterology and Hepatology, Kyoto University Graduate School of Medicine, Kyoto, Japan
}

\section{Key Words \\ Chronic hepatitis C $\cdot$ Hepatocellular carcinoma $\cdot$ Tumor suppressor genes · Chromatin immunoprecipitation}

\begin{abstract}
Chronic hepatitis $\mathrm{C}(\mathrm{CHC})$ triggers oxidative stress and contributes to the emergence of hepatocellular carcinoma (HCC). We previously reported that tumor suppressor gene (TSG) methylation is a critical factor during the early stages of hepatocarcinogenesis. In this study, we clarify the association between oxidative stress and epigenetic alterations during hepatocarcinogenesis. We examined DNA oxidation and methylation profiles in 128 liver biopsy samples from CHC patients. The DNA oxidation and methylated TSG numbers were quantified using immunohistochemical analysis of 8-hydroxydeoxyguanosine (8-OHdG) and quantitative PCR for 11 TSGs, respectively. The quantitative chromatin immunoprecipitation-PCR (ChIP-qPCR) assay in HepG2 and fetal liver $\mathrm{Hc}$ cells treated with $\mathrm{H}_{2} \mathrm{O}_{2}$ was used to quantify trimethyl-H3K4, acetylated-H4K16 (an active chromatin marker), trimethyl-H3K27 (a repressive chromatin marker) and $8-\mathrm{OHdG}$. We analyzed 30 promoters of 25 different
\end{abstract}

TSGs by qPCR. The high levels of 8-OHdG was the only variable that was significantly associated with the increased number of methylated TSGs in CHC $(p<0.0001)$. The ChIPqPCR revealed that after $\mathrm{H}_{2} \mathrm{O}_{2}$ treatment of the cell lines, the 8-OHdG-bound promoters showed a modification from an active chromatin (trimethyl-H3K4 and acetylated-H4K16 dominant) to a repressive chromatin (trimethyl-H3K27 dominant) status. We conclude that oxidative stress alters the chromatin status, which leads to abnormal methylation of TSGs, and contributes to hepatocarcinogenesis in $\mathrm{CHC}$ patients.

(c) 2013 S. Karger AG, Basel

\section{Introduction}

It has been reported that chronic inflammation is a critical factor in the development of cancers such as hepatocellular carcinoma (HCC). Additionally, oxidative stress due to increased production of reactive oxygen species (ROS) plays a pivotal role in carcinogenesis by inducing DNA damage $[1,2]$. These DNA alterations, which activate oncogenes and inactivate tumor suppressor

\section{KARGER}

E-Mail karger@karger.com www.karger.com/ddi
(C) 2013 S. Karger AG, Basel

$0257-2753 / 13 / 0316-0459 \$ 38.00 / 0$
Naoshi Nishida, MD, PhD

Department of Gastroenterology and Hepatology

Kinki University School of Medicine, 337-2 Ohno-higashi

Osakasayama, Osaka 589-8511 (Japan)

E-Mail naoshi@med.kindai.ac.jp 
genes (TSGs), could contribute to the development of cancer in the oxidative stress-affected organs $[3,4]$.

A well-known marker of oxidative DNA damage induced by ROS is 8-hydroxydeoxyguanosine (8-OHdG). A high level of 8-OHdG could be considered as a risk factor in several types of cancer. For example, a higher accumulation of 8-OHdG has been reported in breast, renal and gastric tumor tissues, when compared to adjacent or normal tissues [5-7]. In addition, the cumulative incidence rate of HCC in patients with high 8-OHdG levels in the liver was significantly greater than that in patients with low 8-OHdG levels [8]. Generally, the genotoxic effect of ROS could increase the carcinogenic potential through the induction of base modifications. The formation of $8-\mathrm{OHdG}$ is known to induce $\mathrm{G}>\mathrm{T} / \mathrm{C}>\mathrm{A}$ transversions; unrepaired 8-OHdG causes transversion mutations due to its ability to pair with adenine as well as cytosine bases [9]. However, the recently published whole genome analysis suggests that the mutational spectrum of this type of tumor is heterogeneous [10]. Therefore, it is conceivable that an alternative mechanism for the modulation of cancer-related genes, such as the inactivation of TSGs through epigenetic pathways, might be induced by an increase in 8-OHdG levels.

Previously, we have reported that a number of TSGs, which were abnormally hypermethylated in chronic hepatitis $\mathrm{C}(\mathrm{CHC})$ liver tissues, were strongly and independently associated with an early onset of HCC in patients without any history of HCCs [11]. However, the mechanism responsible for the induction of abnormal methylation of the TSG promoters is still ambiguous. In this study, we addressed this important issue by focusing on the association between the degree of oxidative stress and hypermethylation of TSGs, which is a critical factor in the early stages of human hepatocarcinogenesis. Here, we clearly demonstrate that the generation of ROS and 8-OHdG could play a critical role in the abnormal methylation of TSGs, which in turn should play an important role in the early stages of HCV-related human hepatocarcinogenesis.

\section{Materials and Methods}

\section{Subjects}

For analyzing the relationship between the accumulation of 8-OHdG and hypermethylation of TSGs in CHC, we used the biopsy specimens from $126 \mathrm{CHC}$ subjects, who had no prior history of HCC. The detailed information describing the patient cohort has been described previously [11]. The median age of the patients (25-75\%) was 57 (46-64), and the cohort included 85 male and 43 female subjects. The liver fibrosis stage (F-stage) of each biopsy specimen was determined by the METAVIR scoring system. Among the subjects, 6 cases were scored as $\mathrm{F} 0,40$ were $\mathrm{F} 1,37$ were F2, 23 were F3 and 22 were F4. Informed consent was obtained from each patient and the study was approved by the institutional review boards of the involved institutions.

\section{Immunohistochemical Analyses of 8-OHdG}

We used an archive of liver biopsy specimens, which were fixed in buffered formalin and embedded in paraffin. Sections were stained with hematoxylin and eosin and Masson's trichrome for assessment of morphological changes and the stage of fibrosis. Immunohistochemistry (IHC) staining of 8-OHdG was performed using the avidin-biotin complex method. A mouse monoclonal antibody against 8-OHdG (Japan Institute of the Control of Aging, NIKKEN SEIL Co. Ltd., Tokyo, Japan) was used as described previously [12]. A semiquantitative estimation of the degree of 8-OHdG formation was done by counting the number of stained hepatocyte nuclei. The 8-OHdG levels were classified as strong (50\% or more of total hepatocytes; fig. 1a), moderate (10-49\%; fig. 1b) and weak (9\% or less; fig. 1c). Perls' Prussian blue staining was performed to evaluate the levels of iron deposits. The histologic quantification of hepatic iron was based on the number of hepatocytes showing positive Perls' Prussian blue staining: strong (25\% or more of total hepatocytes; fig. 1d), moderate (5-24\%; fig. 1e), and weak ( $4 \%$ or less; fig. 1f). For both IHC and Perls' Prussian blue staining, ten visual fields from different areas of the liver were used for the evaluation, and 100 nuclei of hepatocytes were counted for each visual field.

\section{Analyses of Abnormal TSG Methylation in Hepatitis Tissue}

The methylation status of the promoters of the 11 TSGs was analyzed in the CHC biopsy specimens. Methylation events of 11 TSGs, HIC-1, GSTP1, SOCS1, RASSF1, CDKN2A, APC, RUNX3, PRDM2, CASP8, CACNA1G and PTGS2 genes were determined. These TSGs were selected because of a unique profile of abnormal methylation in early HCC [11]. For determination of abnormal methylation, we performed quantitative MethyLight assays using the StepOne ${ }^{\mathrm{TM}}$ real-time detection system (Applied Biosystems, Foster City, Calif., USA). The methylation status of 8 TSGs (HIC1, GSTP1, SOCS1, RASSF1, CDKN2A, APC, RUNX3 and $P R D M 2)$ in $\mathrm{CHC}$ specimens were reported previously [11]; PCR primers and probes for these TSGs have already been described. We further examined 3 additional TSGs, CASP8, CACNA1G and PTGS2 in this study. The sequences of PCR primers and TaqMan probes of these 3 TSGs are as follows: the CASP8-forward primer, 5'-AAGTATGTTTAGCGTTCGGGTTTT-3', CASP8reverse primer, $5^{\prime}$-ATACCCAATTTCCAACCATTCAA- $3^{\prime}$, and CASP8-probe, FAM-TTGTACGTTTATGAATTGT-MGB; the CACNA1G-forward primer, 5' -TTTTTTCGTTTCGCGTTTAGGT-3', CACNA1G-reverse primer, 5'-CTCGAAACGACTTCGCCG-3', and CACNA1G-probe, FAM-AAATAACGCCGAATCCGACAACCGA-MGB; the PTGS2-forward primer, 5'-CGGAAGCGTTCGGGTAAAG-3', PTGS2-reverse primer, 5'-AATTCCACCGCCCCAAAC-3', and PTGS2-probe, FAMTTTCCGCCAAATATCTTTTCTTCTTCGCA-MGB. The PCR was performed following the manufacturer's protocol using TaqMan ${ }^{\circledR}$ Fast universal PCR Master Mix (Applied Biosystems), as reported previously [11]. 

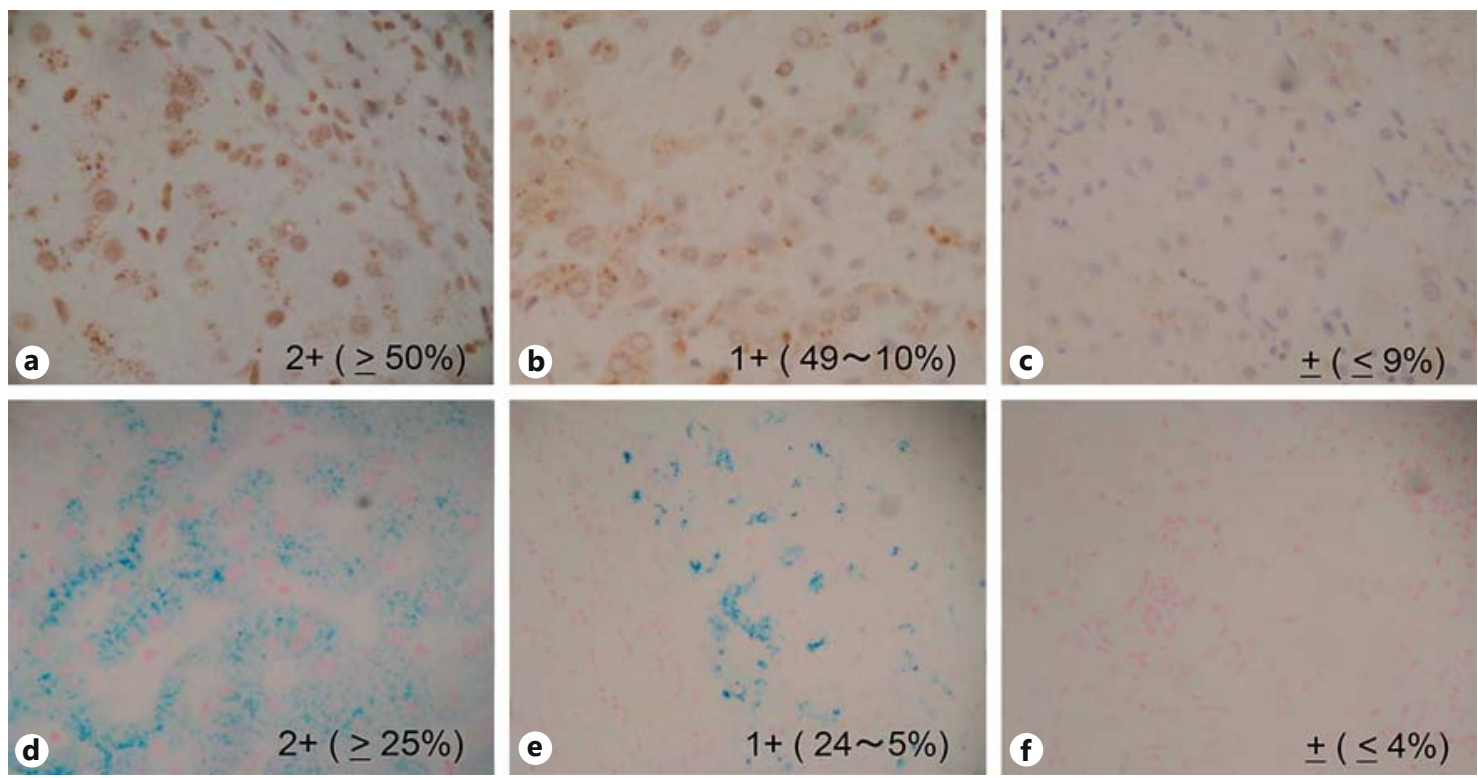

Fig. 1. Immunohistochemical staining of $8-\mathrm{OHdG}(\mathbf{a}-\mathbf{c})$ and iron staining using Perls' Prussian blue (d-f). Among 128 biopsy specimens, 34 showed positive staining of $50 \%$ or more of the total hepatocyte nuclei and were classified as strong $(2+)$ staining $(\mathbf{a})$; similarly, 30 were classified as moderate $(1+)$ staining $(10-49 \%$ of total nuclei; b) and 61 were weak ( \pm ) staining (less than $10 \%$; c). The classification of the specimens based on the hepatic iron deposits were as follows: 10 were classified as strong $(2+)$ deposits $(25 \%$ or more of total hepatocyte; $\mathbf{d}), 40$ were moderate $(1+)$ deposits (5$24 \%$; e) and 75 were shown as weak ( \pm ) deposits (less than $5 \%$; f).
Reagents and Cell Lines for Chromatin Immunoprecipitation

The human HCC cell line, HepG2, was obtained from American Type Culture Collection (Rockville, Md., USA) and human fetal liver Hc cells was purchased from Applied Cell Biology Research Institute (Kirkland, Wash., USA). HepG2 and Hc cells were maintained in Dulbecco's modified Eagle's medium with $10 \%$ fetal bovine serum in a humidified atmosphere with $5 \% \mathrm{CO}_{2}$ at $37^{\circ} \mathrm{C}$. Antibodies for chromatin immunoprecipitation (ChIP) were as follows: $8-\mathrm{OHdG}$ (Japan Institute of the Control of Aging, NIKKEN SEIL Co. Ltd.), acetylated-H4K16 (AcK16H4; Millipore, Billerica, Mass., USA), trimethyl-H3K4 (3MeK4H3), trimethyl-H3K27 (3MeK27H3), panhistone H3 (H3; Wako Pure Chemical Industries Ltd., Osaka, Japan), and rabbit IgG (Genetein Co. Ltd., Tokyo, Japan).

Treatment of HCC Cell Lines with Hydrogen Peroxide, and ChIP before and after Treatment with Hydrogen Peroxide

HepG2 and Hc cells were treated with hydrogen peroxide $\left(\mathrm{H}_{2} \mathrm{O}_{2}\right)$, and ChIP was performed before and after treatment using antibodies against Ack16H4 and 3MeK4H3 (an active histone marker), 3MeK27H3 (a repressive histone marker), 8-OHdG (a DNA damage marker), H3 (ChIP-positive control) and rabbit IgG (ChIP-negative control). In 10-cm culture dishes, $3 \times 10^{6}$ of the cells were seeded $24 \mathrm{~h}$ prior to the experiments. HepG 2 cells were treated with $250 \mu \mathrm{M} \mathrm{H}_{2} \mathrm{O}_{2}$ for $1 \mathrm{~h}$ and $\mathrm{Hc}$ cells were treated with $50 \mu \mathrm{M}$ of $\mathrm{H}_{2} \mathrm{O}_{2}$ for $1 \mathrm{~h}$, respectively. The cells were harvested with trypsin, washed twice and suspended in $0.5 \mathrm{ml}$ of PBS. For the cross-linking of histone and DNA, $13.5 \mu \mathrm{l}$ of $36.6 \%$ (w/v) of formaldehyde was added, and cells were incubated for $8 \mathrm{~min}$ at room temperature. In order to stop the crosslink reaction, $57 \mu \mathrm{l}$ of $1.25 \mathrm{M}$ glycine was subsequently added to the cells; cells were incubated for $5 \mathrm{~min}$ at room temperature and washed with cold PBS. For the ChIP reaction for histone, we used Auto ChIP kit and the SX-8G IP-Star Automated System (Diagenode Inc., Denville, N.J., USA). Chromatin was sheared to a length of 400-800 bp using sonication instruments (Bioruptor $^{\circledR}$ ). For the 8-OHdG ChIP reaction, we used the Auto MeDIP kit (Diagenode Inc.). The chromatin was incubated with antibodies for $10 \mathrm{~h}$, antibody precipitated and eluted from the magnetic beads following the manufacturer's protocol (Diagenode Inc.).

Quantitative ChIP-PCR Analyses of the Promoters of TSGs

Quantitative ChIP-PCR (ChIP-qPCR) of 30 gene promoters of 25 different TSGs was studied to indicate the methylation status in human cancer. qPCR was performed using the EpiScope ${ }^{\circledR}$ Promoter qPCR Array with SYBR Green-based detection (TaKaRa Bio Inc., Otsu, Japan) and the StepOne ${ }^{\mathrm{TM}}$ real-time detection system (Applied Biosystems) according to the manufacturer's protocol. The specificity of targeted PCR product was confirmed by melt-curve analysis, which is essential in an efficient and specific quantitative PCR assay. Alterations in the chromatin associated with damaged DNA (i.e. 8-OHdG-bound DNA elements) before and after the $\mathrm{H}_{2} \mathrm{O}_{2}$ treatment were also assessed. Samples were run in triplicate and data were normalized to amplifications of 5\% input samples. The fold changes in the measures of histone modification and the 8-OHdG level in treated and untreated cells were calculated.

\section{Statistical Analysis}

To determine the significant variables that contribute to an increase in the number of methylated TSGs, the $\chi^{2}$ test was used. Multiple comparisons between the fold changes of AcK16H4, $3 \mathrm{MeK} 4 \mathrm{H} 3,3 \mathrm{MeK} 27 \mathrm{H} 3,8-\mathrm{OHdG}$ and that of $\mathrm{H} 3$ were done using 
Steel's method. The correlation between each fold change (for AcK16H4, 3MeK4H3, 3MeK27H3 and H3) and the 8-OHdG level was evaluated by Spearman's rank correlation test. All p values were calculated employing a two-tailed analysis and $\mathrm{p}<0.05$ was considered statistically significant. All statistical analyses were performed by using JMP version 9.0 software (SAS Institute Inc., Cary, N.C., USA).

\section{Results}

Association between ROS-Mediated DNA Damage and TSG Methylation Events in the Liver during $\mathrm{CHC}$ Infection

In order to evaluate the ROS-mediated DNA damage, we performed IHC staining of 8-OHdG in 125 out of 128 liver biopsy specimens of $\mathrm{CHC}$ tissues. The $\mathrm{CHC}$ cases were categorized into 3 subgroups as described in Materials and Methods. Among the 125 specimens, 34 were classified as having a strong staining $(2+)$ of $8-\mathrm{OHdG}, 30$ as moderate $(1+)$ and 61 as weak $( \pm)$. Similarly, 10 specimens showed strong deposits (2+) of iron, 40 moderate $(1+)$ and 75 weak $( \pm)$. We also assessed TSG promoter methylation in all the $128 \mathrm{CHC}$ biopsy specimens. Of these, $27 \mathrm{CHC}$ specimens showed methylation in 3 or more TSGs, and 101 specimens showed fewer than 3 methylated TSGs.

The association between each clinicopathological factor and the number of methylated TSGs in the liver tissues is summarized in table 1 . Although an increase in the number of methylated TSGs $(\geq 3)$ was more prevalent in $\mathrm{CHC}$ of older-aged males ( $\geq 55$ years) with advanced Fstage fibrosis (F2-F4) and strong deposits of iron, the 8-OHdG level in the liver tissues was the only factor that significantly correlated with the increased TSG methylation in $\mathrm{CHC}(\mathrm{p}<0.0001)$.

\section{Alteration of Histone Modification Induced by $\mathrm{H}_{2} \mathrm{O}_{2}$ Treatment}

The strong relationship between the 8-OHdG level and TSG methylation suggested that the formation of 8-OHdG might induce methylation-associated gene silencing through the formation of repressive histone markers. To test this hypothesis, we used ChIP to measure the fold changes of repressive and active histone markers at the $\mathrm{CpG}$-containing TSG promoters with 8-OHdG before and after $\mathrm{H}_{2} \mathrm{O}_{2}$ treatment. For this purpose, we performed ChIP-qPCR on the promoters of 30 transcripts in the 25 genes using antibodies against AcK16H4 and 3MeK4H3 (the active histone markers), $3 \mathrm{MeK} 27 \mathrm{H} 3$ (the repressive histone marker) and 8-OHdG (to detect damaged DNA elements in the HepG2 cells).
Table 1. Association between the number of methylated TSGs and clinicopathological factors in patients with $\mathrm{CHC}$

\begin{tabular}{|c|c|c|c|c|}
\hline \multirow[t]{2}{*}{ Variables } & \multirow[t]{2}{*}{ Cases, $\mathrm{n}$} & \multicolumn{2}{|c|}{$\begin{array}{l}\text { Methylated } \\
\text { TSGs }^{1}, \mathrm{n}\end{array}$} & \multirow[t]{2}{*}{$\mathrm{p}$ value ${ }^{2}$} \\
\hline & & $<3$ & $\geq 3$ & \\
\hline \multicolumn{5}{|l|}{ Age } \\
\hline$<55$ years & 54 & 46 & 8 & \\
\hline$\geq 55$ years & 74 & 55 & 19 & 0.1311 \\
\hline \multicolumn{5}{|l|}{ Gender } \\
\hline Female & 43 & 38 & 5 & \\
\hline Male & 85 & 63 & 22 & 0.0522 \\
\hline \multicolumn{5}{|l|}{ F-stage } \\
\hline $\mathrm{F} 0$ or $\mathrm{F} 1$ & 46 & 39 & 7 & \\
\hline $\mathrm{F} 2-\mathrm{F} 4$ & 82 & 62 & 20 & 0.2135 \\
\hline \multicolumn{5}{|c|}{ Staining of $8-\mathrm{OHdG}$} \\
\hline \pm & 61 & 58 & 3 & \\
\hline $1+$ & 30 & 22 & 8 & \\
\hline $2+$ & 34 & 20 & 14 & $<0.0001$ \\
\hline \multicolumn{5}{|l|}{ Iron deposit } \\
\hline \pm & 75 & 63 & 12 & \\
\hline $1+$ & 40 & 31 & 9 & \\
\hline $2+$ & 10 & 6 & 4 & 0.2191 \\
\hline
\end{tabular}

${ }^{1}$ The number of methylated TSGs was determined using MethyLight. Eleven TSGs (HIC-1, GSTP1, SOCS1, RASSF1, CDKN2A, $A P C, R U N X 3, P R D M 2, C A S P 8, C A C N A 1 G$ and PTGS2) were analyzed. ${ }^{2} \mathrm{p}$ values were calculated using the $\chi^{2}$ test.

Among the promoters of the 30 transcripts, we successfully amplified the promoter regions of 29 transcripts using the post-ChIP DNA for the measurement of AcK16H4. Similarly, 25, 27 and 26 promoters could be amplified after the ChIP for 3MeK4H3, 3MeK27H3 and 8 -OHdG, respectively. All but one promoter (the LOX gene) were amplified after the ChIP for H3. Moreover, 23 promoters were amplified by all 4 ChIP-qPCRs, and the fold changes of the ChIP-qPCR values were determined for these loci using $\mathrm{H}_{2} \mathrm{O}_{2}$-treated and untreated cells (fig. 2a). In the rabbit IgG-negative control ChIP assay, 10 of the 30 gene promoters were slightly amplified; however, the fold changes for the negative control could not be calculated because of its low levels. The median values $(25-75 \%)$ of fold changes were $0.70(0.55-0.90)$ for AcK16H4, $0.80(0.60-1.10)$ for $3 \mathrm{MeK} 4 \mathrm{H} 3,1.60(1.30-1.80)$ for $3 \mathrm{MeK} 27 \mathrm{H} 3,1.45(1.00-$ 1.93) for 8-OHdG and $1.10(0.90-1.40)$ for H3. For 17 promoters, the $8-\mathrm{OHdG}$ fold changes were more than 1.0 , suggesting the increase of $8-\mathrm{OHdG}$ at $\mathrm{CpG}$ islandcontaining TSG promoters after $\mathrm{H}_{2} \mathrm{O}_{2}$ treatment. Simi- 
Fig. 2. Alteration of histone modification and its association with 8-OHdG levels after $\mathrm{H}_{2} \mathrm{O}_{2}$ treatment at the $\mathrm{CpG}$ island-containing promoters of TSGs. a HepG2 cells were either treated with or without $250 \mu \mathrm{M}$ of $\mathrm{H}_{2} \mathrm{O}_{2}$ for $1 \mathrm{~h}$, followed by ChIP for AcK16H4, 3MeK4H3 as an active histone marker, $3 \mathrm{MeK} 27 \mathrm{H} 3$ as a repressive histone marker, 8-OHdG, positive control of $\mathrm{H} 3$ or negative control of rabbit IgG. The y-axis indicates the fold changes of ChIP-qPCR values over $\mathrm{H} 3$ values between the treated and untreated samples. $\mathrm{T} / \mathrm{U}=$ Ratio of PCR values of treated/untreated cells. ${ }^{*}$ Indicates the splice variants of the corresponding gene. $\mathbf{b}$ Comparisons of the fold changes indicated by ChIP-qPCR between the IP of each histone modification or $8-\mathrm{OHdG}$ and that of $\mathrm{H} 3$. p values were calculated using Steel's method for nonparametric multiple comparison.

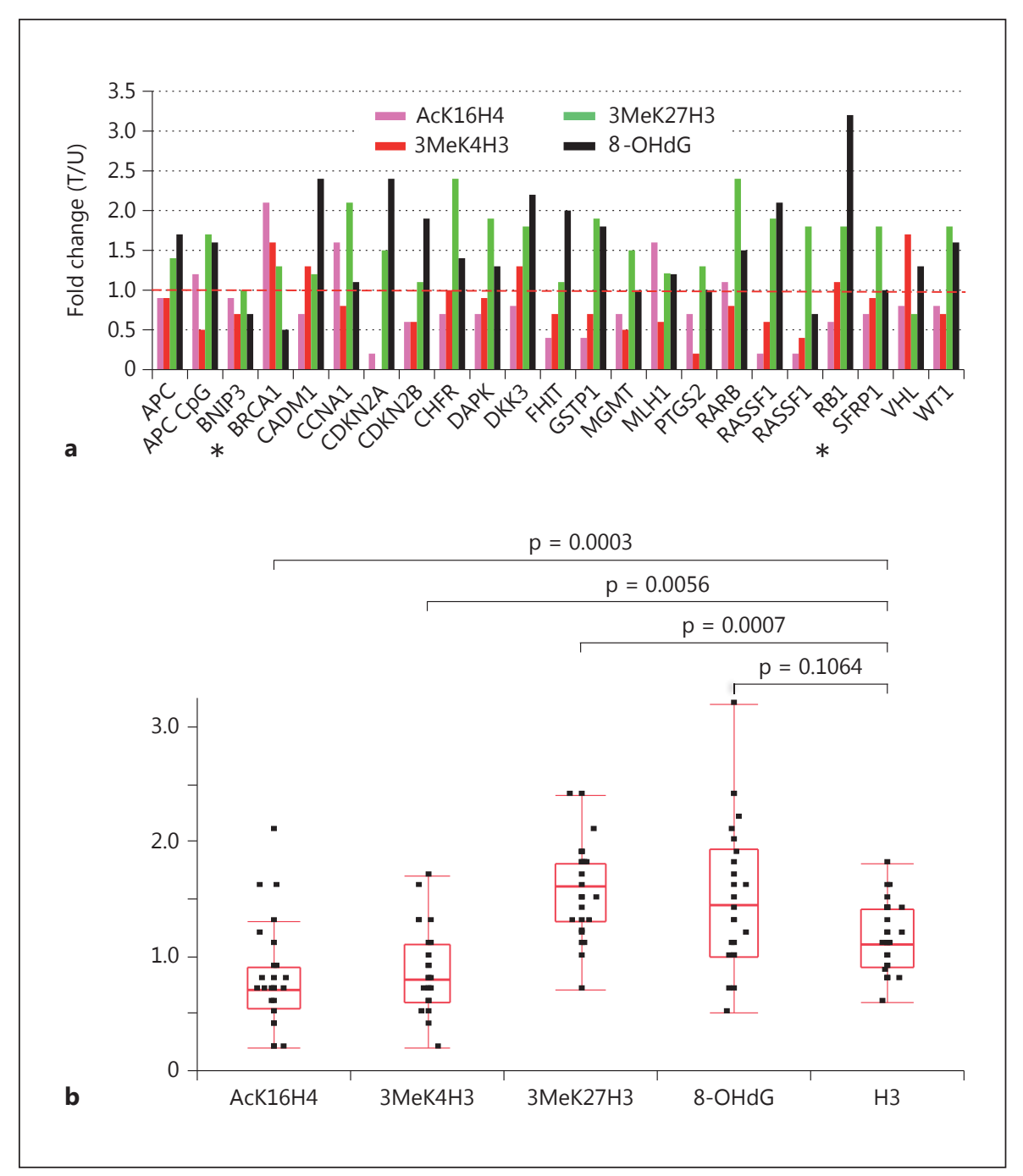

larly, the fold changes of the recessive histone marker $3 \mathrm{MeK} 27 \mathrm{H} 3$ were greater than 1.0 for $21 \mathrm{TSG}$ promoters. On the other hand, there was a reduction of the active histone markers Ack16H4 and 3MeK4H3 for 17 and 15 promoters, respectively, after treatment with $\mathrm{H}_{2} \mathrm{O}_{2}$ (fig. 2a). The correlation between the fold changes of AcK16H4, 3MeK4H3, 3MeK27H3, H3 and that of 8-OHdG were also analyzed (fig. 2b). Although it was not statistically significant, the 8 -OHdG fold change comparatively increased to that of $\mathrm{H} 3$ ( $\mathrm{p}=0.1064$ by Steel's method for nonparametric multiple comparison; fig. 2b). The AcK16H4 and 3MeK4H3 fold change values were significantly lower, and the fold change values for $3 \mathrm{MeK} 27 \mathrm{H} 3$ were significantly higher than that of $\mathrm{H} 3$ $(\mathrm{p}=0.0003,0.0056$ and 0.0007 for AcK16H4, 3MeK4H3 and $3 \mathrm{MeK} 27 \mathrm{H} 3$, respectively, by Steel's multiple comparison test).

We further examined the relationship between the fold changes of $8-\mathrm{OHdG}$ and active and recessive histone markers using $\mathrm{H}_{2} \mathrm{O}_{2}$-treated and untreated fetal liver Hc cells. Although the correlation was not prominent for AcK16H4 ( $r=-0.2789, p=0.1507$; fig. 3a), there was a moderate inverse correlation between the fold changes of $8-\mathrm{OHdG}$ and those of active histone markers $(r=-0.5273, p=0.0039$ for $3 \mathrm{MeK} 4 \mathrm{H} 3$; fig. $3 b)$. Interestingly, a strong correlation was detected between the fold changes of 8-OHdG and those of recessive histone markers of $3 \mathrm{MeK} 27 \mathrm{H} 3(\mathrm{r}=0.7605, \mathrm{p}<0.0001$; fig. 3c). As expected, no correlation was observed between the fold changes of $8-\mathrm{OHdG}$ and those of $\mathrm{H3}$ 


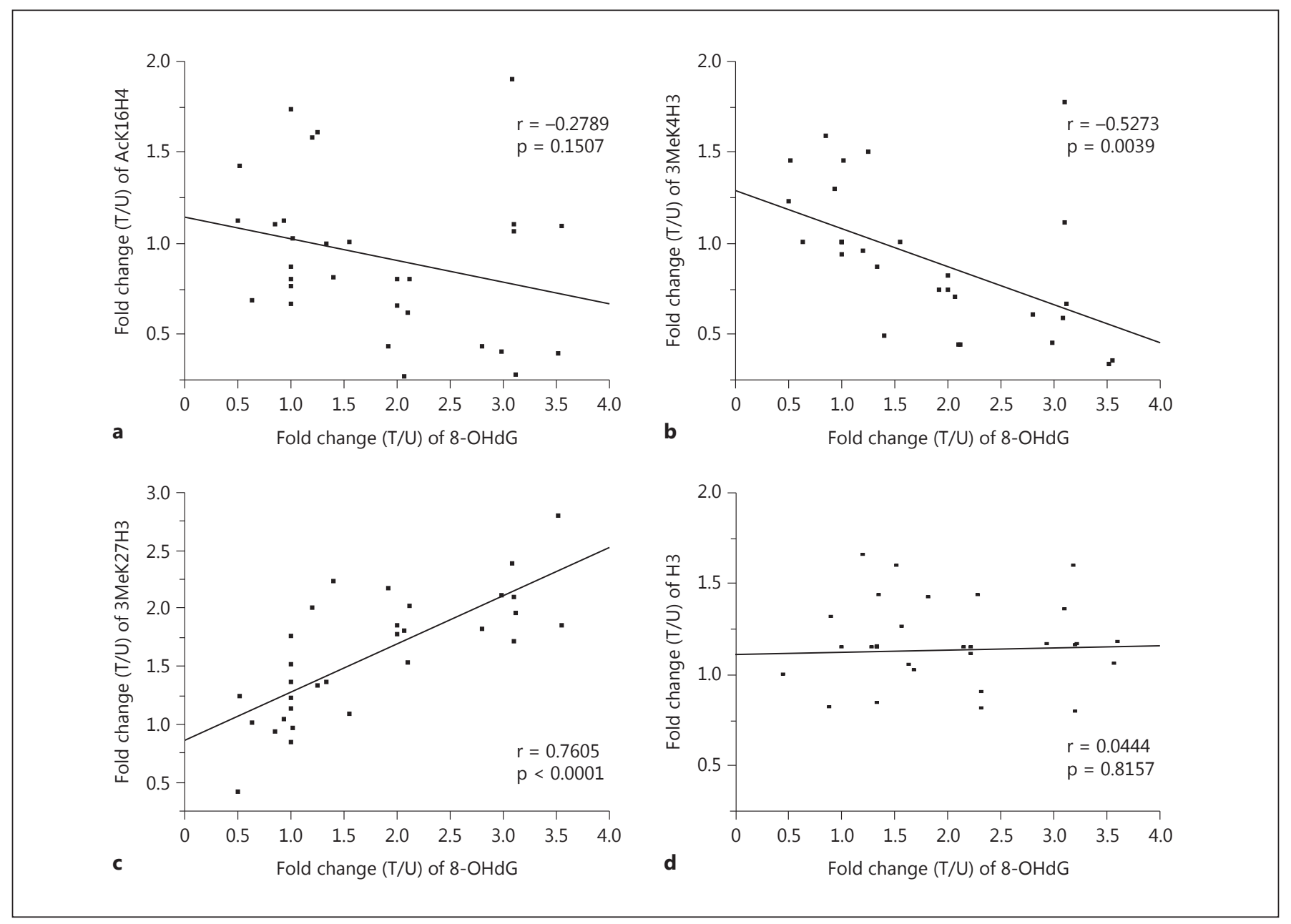

Fig. 3. Correlations between the fold changes of ChIP-qPCR values for histone and 8-OHdG. Correlations between the fold changes of AcK16H4 and 8-OHdG (a), 3MeK4H3 and 8-OHdG (b), 3MeK27H3 and 8-OHdG (c), and H3 and 8-OHdG (d). Correlations between each fold change and that of $8-\mathrm{OHdG}$ were evaluated by Spearman's rank correlation test.

(fig. 3d). These findings also indicated that the decrease in active chromatin and the increase in repressive chromatin take place on the 8-OHdG-enriched CpG islandcontaining TSG promoters, after $\mathrm{H}_{2} \mathrm{O}_{2}$ treatment.

\section{Discussion}

A number of reports suggested that the activation of oncogenes and inactivation of TSGs was a characteristic feature of human cancers, including HCC $[3,4]$. We previously reported that the inactivation of TSGs through promoter methylation was more prevalent in $\mathrm{HCV}$-related HCCs than in HCV-negative tumors [13]. On the other hand, it was also reported that oxidative stress could play a central role in the pathogenesis of $\mathrm{CHC}$, and could increase the risk of HCC development $[13,14]$. In this study, we examined whether persistent stimulation by ROS and subsequent DNA damage, indicated by the formation of 8-OHdG, could increase the risk for HCC development through the induction of epigenetic instability in hepatocytes. Here, we demonstrated that the 8-OHdG level was the only factor associated with an increased number of methylated TSGs in the liver of $\mathrm{CHC}$, and that TSGs carrying higher levels of 8-OHdG facilitated the modification of the active chromatin to a repressive form after stimulation by ROS.

So far, several reports have suggested that increased ROS production is observed in $\mathrm{CHC}$ and the amount of ROS is associated with the onset of HCC $[8,14,15]$. Ac- 
tivation of oxidative stress pathways in noncancerous human liver tissue reportedly predicted the recurrence of HCC in patients who underwent a hepatectomy [16]. ROS could also induce genetic alterations such as point mutations because oxidative DNA damage, indicated by $8-\mathrm{OHdG}$, could induce DNA base mutations such as $\mathrm{G}>\mathrm{T} / \mathrm{C}>\mathrm{A}$ transversions [9]. However, although this type of base mutation is commonly found in HCC, whole genome and exome analyses revealed that the mutational spectrum of HCC is heterogeneous [10]. In addition, so far, the frequencies of common mutations in specific TSGs in HCC were not high; the frequencies of the mutations were around 30 and $15 \%$ for $p 53$ and $\beta$-catenin, respectively [17-21]. On the other hand, a considerable number of cancer-related genes, such as the CDKN2A, RASSF1A, GSTP1 and APC, showed an alteration in the methylation status in HCC $[13,21]$. Regional hypermethylation of the gene promoters leads to transcriptional inactivation of the corresponding TSGs and hypomethylation could cause increased expression of oncogene and transposable DNA elements, both of which could contribute to carcinogenesis.

Previously, we selected the TSGs that showed a high level of methylation in early HCC, and reported that methylation of these TSGs was a unique marker for earlystage HCV-related HCC [11]. In addition, we found that a number of these methylated TSGs in the liver were significantly associated with the onset of HCC in patients with $\mathrm{CHC}$ without a prior history of HCC [11]. In this study, we further clarified that the level of 8-OHdG in the liver was the only factor that showed significant association with the methylated TSG number in the same CHC cohort. Previous reports suggested that the treatment of the $\mathrm{HCC}$ cell line by $\mathrm{H}_{2} \mathrm{O}_{2}$ induced an increase in Snail expression and hypermethylation of the E-Cadherin promoter. Snail was also shown to recruit HDAC1 and DNMT1 to the E-Cadherin gene [22]. Another report showed that oxidative stress induced by $\mathrm{H}_{2} \mathrm{O}_{2}$ recruits DNA methyltransferase 1 (DNMT1) to damaged chromatin in colon cancer cell lines [23]. It resulted in relocalization of the polycomb repressive protein complex from non-GC-rich to GC-rich areas [23]. Thus, it was reasonable to speculate that oxidative damage led to the formation and relocalization of a polycomb repressive complex, which may explain cancer-specific aberrant DNA methylation and transcriptional silencing of TSGs. To explore this possibility, we treated HCC-derived cell lines and fetal liver cells with $\mathrm{H}_{2} \mathrm{O}_{2}$, and determined the effect of ROS on the modulation of histone. As expected, treatment with $\mathrm{H}_{2} \mathrm{O}_{2}$ induced the formation of 8-OHdG on the CpG island-containing TSGs. Interestingly, after treatment the measurement of the repressive histone marker, $3 \mathrm{MeK} 27 \mathrm{H} 3$, increased, and that of the active histone markers, AcK16H4 and $3 \mathrm{MeK} 4 \mathrm{H} 4$, decreased. It was well known that the polycomb complex could induce repressive histone markers such as $3 \mathrm{MeK} 27 \mathrm{H} 3$. The repressive histone marker was associated with the induction of DNA methylation in the corresponding region. On the other hand, our results also indicated that the 8-OHdG fold change using $\mathrm{H}_{2} \mathrm{O}_{2}$-treated and untreated cells was clearly correlated to that of $3 \mathrm{MeK} 27 \mathrm{~K} 3$, and inversely correlated to that of AcK16H4 and $3 \mathrm{MeK} 4 \mathrm{H} 4$. These facts support the idea that ROS induced an alteration of histone modification to the repressive form at the CpG island-containing TSG promoters and induced hypermethylation through the formation of $8-\mathrm{OHdG}$, which could lead to inactivation of TSGs by epigenetic mechanisms.

In this study, we clearly demonstrated that the ROSinduced DNA damage could cause the induction of repressive histone markers on TSG promoters. Therefore, it is reasonable to believe that the increased TSG methylation seen in HCC could be reflective of the alteration of chromatin status induced by ROS. As the accumulation of methylated TSGs in CHC tissues has been shown to be a strong risk factor for the early onset of HCC, ROS probably plays a critical role in human hepatocarcinogenesis through the induction of epigenetic instability and regional hypermethylation in $\mathrm{CHC}$.

\section{Acknowledgements}

This work was supported in part by Grant-in-Aid for Scientific Research (KAKENHI: 24590997) from the Japanese Society for the Promotion of Science (N. Nishida) and a grant from the Smoking Research Foundation (N. Nishida).

\section{Disclosure Statement}

The authors have no conflicts of interest to disclose.

References

\footnotetext{
1 Stauffer JK, Scarzello AJ, Jiang Q, Wiltrout RH: Chronic inflammation, immune escape, and oncogenesis in the liver: a unique neighborhood for novel intersections. Hepatology 2012;56:1567-1574.

2 Kim DY, Han K: Epidemiology and surveillance of hepatocellular carcinoma. Liver Cancer $2012 ; 1: 2-14$.
} 
- 3 Nishida N: Impact of hepatitis virus and aging on DNA methylation in human hepatocarcinogenesis. Histol Histopathol 2010;25:647654.

4 Nishida N, Goel A: Genetic and epigenetic signatures in human hepatocellular carcinoma: a systematic review. Curr Genomics 2011; 12:130-137.

5 Okamoto K, Toyokuni S, Uchida K, Ogawa O, Takenewa J, Kakehi Y, Kinoshita H, HattoriNakakuki Y, Hiai H, Yoshida O: Formation of 8-hydroxy-2'-deoxyguanosine and 4-hydroxy-2-nonenal-modified proteins in human renal-cell carcinoma. Int J Cancer 1994; 58:825-829.

6 Musarrat J, Arezina-Wilson J, Wani AA: Prognostic and aetiological relevance of 8-hydroxyguanosine in human breast carcinogenesis. Eur J Cancer 1996;32A:1209-1214.

7 Lee BM, Jang JJ, Kim HS: Benzo[a]pyrene diol-epoxide-i-DNA and oxidative DNA adducts associated with gastric adenocarcinoma. Cancer Lett 1998;125:61-68.

8 Chuma M, Hige S, Nakanishi M, Ogawa K, Natsuizaka M, Yamamoto Y, Asaka M: 8-hydroxy-2'-deoxy-guanosine is a risk factor for development of hepatocellular carcinoma in patients with chronic hepatitis $\mathrm{C}$ virus infection. J Gastroenterol Hepatol 2008;23:14311436.

9 Unfried K, Schurkes C, Abel J: Distinct spectrum of mutations induced by crocidolite asbestos: clue for 8-hydroxydeoxyguanosinedependent mutagenesis in vivo. Cancer Res 2002;62:99-104.

10 Nishida N, Kudo M: Recent advancements in comprehensive genetic analyses for human hepatocellular carcinoma. Oncology 2013; 84(suppl 1):93-97.
11 Nishida N, Kudo M, Nagasaka T, Ikai I, Goel A: Characteristic patterns of altered DNA methylation predict emergence of human hepatocellular carcinoma. Hepatology 2012;56: 994-1003.

12 Toyokuni S, Tanaka T, Hattori Y, Nishiyama Y, Yoshida A, Uchida K, Hiai H, Ochi H, Osawa T: Quantitative immunohistochemical determination of 8-hydroxy-2'-deoxyguanosine by a monoclonal antibody n45.1: its application to ferric nitrilotriacetate-induced renal carcinogenesis model. Lab Invest 1997;76: 365-374.

13 Nishida N, Nagasaka T, Nishimura T, Ikai I, Boland CR, Goel A: Aberrant methylation of multiple tumor suppressor genes in aging liver, chronic hepatitis, and hepatocellular carcinoma. Hepatology 2008;47:908-918.

-14 Tanaka S, Miyanishi K, Kobune M, Kawano Y, Hoki T, Kubo T, Hayashi T, Sato T, Sato Y, Takimoto R, Kato J: Increased hepatic oxidative DNA damage in patients with nonalcoholic steatohepatitis who develop hepatocellular carcinoma. J Gastroenterol 2013, E-pub ahead of print.

15 Maki A, Kono H, Gupta M, Asakawa M, Suzuki T, Matsuda M, Fujii H, Rusyn I: Predictive power of biomarkers of oxidative stress and inflammation in patients with hepatitis $\mathrm{C}$ virus-associated hepatocellular carcinoma. Ann Surg Oncol 2007;14:1182-1190.

16 Tanaka S, Mogushi K, Yasen M, Ban D, Noguchi N, Irie T, Kudo A, Nakamura N, Tanaka H, Yamamoto M, Kokudo N, Takayama T, Kawasaki S, Sakamoto M, Arii S: Oxidative stress pathways in noncancerous human liver tissue to predict hepatocellular carcinoma recurrence: a prospective, multicenter study. Hepatology 2011;54:1273-1281.

17 Nishida N, Fukuda Y, Kokuryu H, Sadamoto $\mathrm{T}$, Isowa G, Honda K, Yamaoka Y, Ikenaga M, Imura H, Ishizaki K: Accumulation of allelic loss on arms of chromosomes 13q, 16q and $17 \mathrm{p}$ in the advanced stages of human hepatocellular carcinoma. Int J Cancer 1992;51:862868.
18 Nishida N, Fukuda Y, Kokuryu H, Toguchida J, Yandell DW, Ikenega M, Imura H, Ishizaki $\mathrm{K}$ : Role and mutational heterogeneity of the p53 gene in hepatocellular carcinoma. Cancer Res 1993;53:368-372.

19 Nishida N, Fukuda Y, Komeda T, Kita R, Sando T, Furukawa M, Amenomori M, Shibagaki I, Nakao K, Ikenaga M, et al: Amplification and overexpression of the cyclin D1 gene in aggressive human hepatocellular carcinoma. Cancer Res 1994;54:3107-3110.

20 Nishida N, Fukuda Y, Ishizaki K, Nakao K: Alteration of cell cycle-related genes in hepatocarcinogenesis. Histol Histopathol 1997;12: 1019-1025.

21 Nishida N, Nishimura T, Nagasaka T, Ikai I, Goel A, Boland CR: Extensive methylation is associated with $\beta$-catenin mutations in hepatocellular carcinoma: evidence for two distinct pathways of human hepatocarcinogenesis. Cancer Res 2007;67:4586-4594.

22 Lim SO, Gu JM, Kim MS, Kim HS, Park YN, Park CK, Cho JW, Park YM, Jung G: Epigenetic changes induced by reactive oxygen species in hepatocellular carcinoma: Methylation of the E-cadherin promoter. Gastroenterology 2008;135:2128-2140.

23 O'Hagan HM, Wang W, Sen S, Destefano Shields C, Lee SS, Zhang YW, Clements EG, Cai Y, Van Neste L, Easwaran H, Casero RA, Sears CL, Baylin SB: Oxidative damage targets complexes containing DNA methyltransferases, SIRT1, and polycomb members to promoter CpG islands. Cancer Cell 2011;20:606619 . 